\title{
Terahertz responsivity of field-effect transistors under arbitrary biasing conditions
}

Péter Földesy 1,2,a)

${ }^{1}$ Computer and Automation Research Institute, Hungarian Academy of Sciences, Budapest, 1111 Hungary

${ }^{2}$ Pázmány Péter Catholic University, Faculty of Information Technology, Budapest, 1083 Hungary

Current biased photoresponse model of long channel field-effect transistor (FET) detectors is introduced to describe the low frequency behavior in complex circuit environment. The model is applicable in all FET working regions, including subthreshold, linear, saturated modes, includes bulk potential variations, and handles the simultaneous gate-source and drain-source detection or source-driven topologies. The model is based on the phenomenological representation that links the photoresponse to the gate transconductance over drain current ratio $\left(g_{m} / I_{D}\right)$ and circuit theory. A derived method is provided to analyze the detector behavior, to characterize existing antenna coupled detectors, and to predict the photoresponse in a complex circuit. The model is validated by measurements of $180 \mathrm{~nm}$ gate length silicon and GaAs high electron mobility (HEMT) FET.

\section{INTRODUCTION}

It is widely exploited that terahertz FET detectors give higher response in a non-open drain configuration, when current is forced through the channel [1][2][3][4][5][6]. However, applying bias current to the detector often show significant differences from their expected behavior. Theoretical works on two dimensional electron gases (2DEG) showed that resonant detection can yield high gain by 
proper boundary conditions regarding both gated [1] and ungated [2] structures, including current bias caused asymmetry. In non-resonant detection, the same theory states that a small current increases sensitivity. The explanation is that the reduced charge concentration near the drain terminal becomes more sensitive to the high frequency perturbation when the transistor operation mode shifts from linear to the saturation region [7]. Among the existing technologies (e.g. GaAs, GaN, Si, SOI) the Si based standard CMOS planar technology provides actually the most matured solution for building focal plane arrays [8][9][10]. The first models regarding MOS transistors as direct power detectors and mixers date back to the ' 80 -ies [11]. An extended model was given that described the higher frequencies as well by non quasi-static approximation [12]. That was followed by other complementary works, e.g. [3]. The work of Sakowicz et al. [13] has introduced a phenomenological model, which is applicable under broad operation conditions. This model covers the non-resonant detection of FET detectors and describes the open drain behavior in the presence of instrumentation load. The advantage of this model is that relates the photoresponse to the DC channel conductivity, which is a measurable quantity or could be simulated by arbitrary electronic models. However the effect of source-drain non zero current is not covered by the model. Another interesting phenomenon of the current biased operation is the sign change of the measured photoresponse. The physical explanations of the negative response were mentioned in [1] and [14] also describes a possible reason for the phenomenon. Guttin el al. [15] described a SPICE model based on the nonlinear power detector model for circuit modeling. But, the article does not address the effect of the nonzero source-drain current. T. A. Elkhatib et al. [4] introduced a phenomenological model to describe ohmic and in the strong saturation regimes. The model interpolates the saturation current related solution of D. Veksler et al. [1] with a simplified saturation FET model consisting of the channel length modulation parameter and the threshold voltage dependence on the source-bulk potential to model the deep saturation behavior. Beside the higher recorded responsivity of the current biased FET 
detectors, the measured noise spectral density increases dramatically along with the applied bias current. Both theoretical and practical improvement of the signal-to-noise ratio (SNR) in silicon FETs is analyzed thoroughly by A. Lisauskas el at. [5]. The used hydrodynamic transport model predicts only slight improvement in the subthreshold conditions, which has not been proved by practical measurements due to the other deteriorating noise factors.

The above mentioned models explain the measured characteristics under specific source-drain biasing conditions supposing various architectural restrictions, such as the transistor source and bulk terminals are connected to ground potential or only the gate-source terminal is coupled to the radiation. The introduced model describes the FET detector behavior under non zero source-drain current in all FET working domain - including depletion, strong and weak inversion, effect of bulk potential - keeping high correlation with the measurement results and explain the apparent amplification and response sign change.

\section{MODEL}

The developed model is valid under the following conditions: non-resonant detection conditions and long channel, symmetric device. That is, the product of the used frequency $\left(\omega_{A C}\right)$ and the inverse momentum relaxation time of the electrons $(\tau)$ is smaller than unity $\omega_{A C} \tau \ll 1$. Hence, the plasmons are overdamped in the channel and the effective signal penetration depth $\left(l_{e f f}\right)$ from the source/drain is smaller than the actual channel length $\left(l_{e f f} \ll L\right)$. Consequently, the photoresponse is generated near the source and drain terminals and the quasi-static distributed RLC or wavequide channel description is applicable. The situation of the more practical case when $l_{e f f}$ is less but comparable to the channel's length is also investigated later on. As an outcome, the source-gate and drain-gate AC mixing terms are the dominant in the photoresponse and no drain-source mixing terms appears. Last, the detector has a 
symmetric physical structure, and the source and drain terminals can be swapped. Though these conditions restrict the applicability of the model, still the selected domain is the principal in sensing and imaging practice (e.g. [8][9][16][17]).

First, the radiation coupling efficiency is formalized, and then the open drain or unloaded response is revised. The case of source drain current is described as a potential difference between the two terminals, which leads to the separated photoresponse model of the source and the drain. Then, the incircuit behavior is analyzed, and finally measurement examples are presented.

\section{A. Radiation coupling}

The incident radiation power received by the antenna and routed to the drain or source terminals are not necessary equal in magnitude to that appears on the gate terminal: the antenna structure can be asymmetric; a terminal connection is not perfect $\mathrm{AC}$ ground; the focusing on a large device varies; and the unshielded wiring receives radiation as well. In addition to the multiple terminal coupled detectors, a source-driven (similarly drain-driven) approach simplified the radiation coupling described in [18][19]. This approach connects the source terminal only to an antenna and provides AC ground to the other terminals. There are also experimental results of gate-driven, or gate only coupled, detection in [20] as well. Hence, a descriptive model must take into consideration that the RF signal is fed to the source, gate, and drain terminals simultaneously with potentially different coupling efficiency. In order to describe the possible alteration from the ideal case of perfect antenna and no parasitic coupling, we introduce efficiency factors. These efficiencies are denoted by $\eta_{S}, \eta_{D}, \eta_{G}$ for the source, drain, and gate respectively. The electric field than is expressed from the incident RF power $P_{a c}$ as:

$$
\left[\begin{array}{l}
u_{a c}^{S} \\
u_{a c}^{D} \\
u_{a c}^{G}
\end{array}\right]=\left[\begin{array}{l}
\eta_{S} \\
\eta_{D} \\
\eta_{G}
\end{array}\right] \sqrt{k P_{a c}}
$$


where $k$ represents a normalization factor of resistance dimension so as $0 \leq \eta_{S, D, G} \leq 1$. The corresponding model can be seen in FIG. 1.

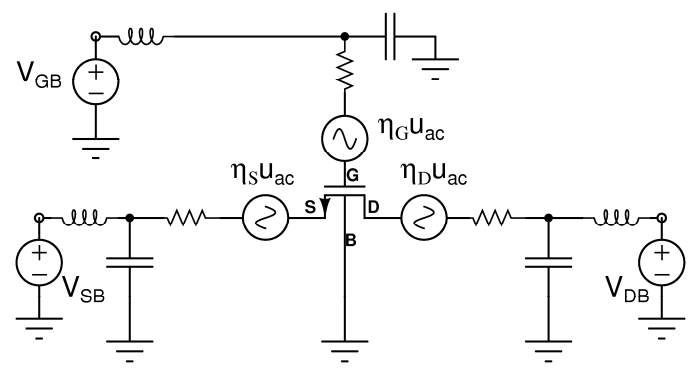

FIG. 1. Illustration of the FET detector notation and the coupling efficiencies. The arrows indicate the antenna connection.

Combining that the FETs behave as square-law power detectors [8] and the model preassumptions of non-resonant detection and long channel approximation, the resulting response becomes different on the terminals depending on the in-situ conditions of $u_{a c}$ signal amplitude and coupling efficiencies:

$$
V_{r}^{G S(D)} \propto u_{a c}^{S(D)} u_{a c}^{G}=\eta_{\mathrm{S}(\mathrm{D})} \eta_{\mathrm{G}} k P_{a c}
$$

The common situation usually suppose ideal situation such as $\eta_{D, G}=1, \eta_{S}=0$. In case of the source-, drain-, or gate-driven approaches, the photoresponse remains quadratic to the electric field [19] and the terminal response can be described as $V_{r}^{G S(D)} \propto\left\{u_{a c}^{S(D, G)}\right\}^{2}=\eta_{\mathrm{S}(\mathrm{D}, \mathrm{G})} k P_{a c}$. Finally, the intrinsic response is defined as $V_{r}^{0}=k P_{a c}$, and $\eta_{\mathrm{G}}=1$ is used in order to unify the description of the different coupling approaches and in order to distinguished from the measurable extrinsic value.

\section{B. Open drain response}

Several models have been founded based on fluidic approximation of the channel 2DEG by [7] [12]. These models rely on solving the continuity equation with the motion counterpart at appropriate boundary conditions. These models explain, besides the power detector like photoresponse, the homodyne and heterodyne mixing, though the derived models are not valid in weak inversion or in 
depletion. This limitation also holds for the transmission line models of the 2DEG channel [14]. In [19] the unified charge control model served as a basis of the rectification, describing the process in subthreshold operational region as well. Sakowicz et al. [13] introduced a description of the open drain non-resonant photoresponse as a function of the channel conductance. This approach is applicable in the subthreshold region as well and connects the measurable DC transfer characteristics with the photoresponse in a simple and elegant way. In the model building, we used this model as it covers broad range of operational conditions. The response $V_{r}^{0}$ is estimated by [13] the channel conductivity $\left(\sigma_{s}\right)$ and the small current DC current transfer function as:

$$
V_{r}^{0} \propto P_{a c} \frac{\partial}{\partial V_{G}} \ln \left(\sigma_{S}\right) \propto P_{a c} \frac{\partial}{\partial V_{G}} \ln \left[I_{D S}\left(V_{G S}\right)\right]
$$

Note, that this equation is equal to the transconductance-to-current-ratio:

$$
\frac{\partial}{\partial V_{G}} \ln \left[I_{D S}\left(V_{G S}\right)\right]=\frac{1}{I_{D S}\left(V_{G S}\right)} \frac{\partial I_{D S}\left(V_{G S}\right)}{\partial V_{G}}=\frac{g_{m}}{I_{D S}\left(V_{G S}\right)}
$$

The $g_{m} / I_{D}$ is an important figure of merit and tool of analog low voltage, low power design [21]. The maximum $g_{m} / I_{D}$ ratio appears in weak inversion and is equal to $1 / n V_{t h}$, where $n$ is the slope factor and $V_{t h}$ the thermal voltage. On the lower gate-source voltage region, with exponentially decreasing sourcedrain current, the $g_{m} / I_{D}$ quickly falls as the leakage currents become dominant in the $I_{D}$ [22]. FIG. 2 compares the open drain resistively loaded photoresponse of the later detailed silicon sample with the $g_{m} / I_{D}$ based Sakowicz et al. [13], the transmission-line based Preu et al. [14] models. 


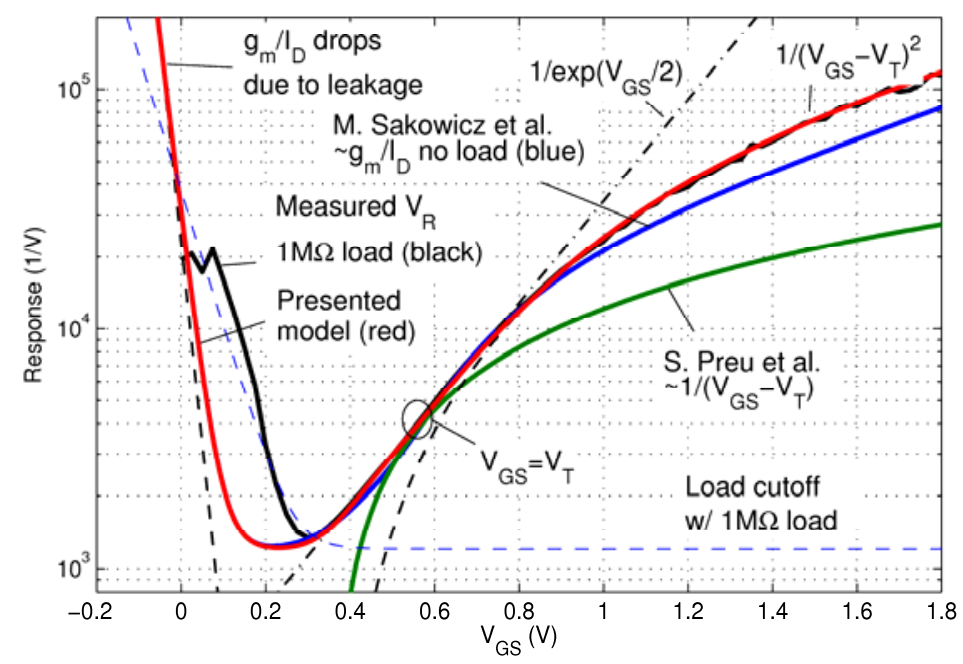

FIG. 2. (Color online) Comparison of different models of the open drain photoresponse silicon FET detector under no load and with $1 \mathrm{M} \Omega$ drain load.

\section{Nonzero source drain current}

As a next step, the situation of actual source drain current $\left(V_{D S} \neq 0\right)$ is analyzed. Under the model assumptions, the DC photoresponse depends only on the near terminal conditions, namely the RF signal and the channel's carrier density at the terminals. As exact analytical solution does not exist for the inversion charge density as a function of the applied voltage, the measurable transfer functions is linked to the photoresponse by first applying an analytical circuit model and then highlight a practical measurement method to avoid model assumptions.

The EPFL-EKV FET model [23][24] model has been chosen as it has physics-based analytical solution for the drain current that is related to the channel inversion charge $\left(Q_{i n v}\left(V_{c h}\right)\right)$. The drain current is derived from the inversion charge density $\left(\rho_{s}\right)$ at the source and drain ends of the channel by decomposing the current to a forward $\left(I_{F}\right)$ and reverse currents $\left(I_{R}\right)$ :

$$
I_{D S}=\beta \int_{V_{S}}^{\infty}\left[-\frac{Q_{i n v}\left(V_{c h}\right)}{C_{o x}}\right] d V_{c h}-\beta \int_{V_{D}}^{\infty}\left[-\frac{Q_{i n v}\left(V_{c h}\right)}{C_{o x}}\right] d V_{c h}
$$




$$
I_{D S}=I_{F}-I_{R}
$$

The inversion charge is estimated along the channel as [25]:

$$
\rho_{S}(x)=2 \rho_{0} \ln \left\{1+0.5 \exp \left(\frac{V_{G S}-V_{T}-\alpha V_{F}(x)}{\eta V_{t h}}\right)\right\}
$$

with $\mathrm{V}_{\mathrm{F}}$ being the quasi-Fermi potential of the channel depending on $V_{D S}, V_{t h}$ the thermal voltage, $\alpha$ the bulk effect parameter close to unity, $\rho_{0}=\eta V_{t h} C_{o x} / q$ the density of minority carriers per unit area at threshold voltage, $\eta$ the subthreshold ideality factor, $V_{T}$ the FET threshold voltage, $0 \leq x \leq L$, and $C_{o x}$ the gate capacitance per unit area. The forward and reverse currents are usually expressed as normalized currents $\left(i_{f}, i_{r}\right)$ multiplied by the so called specific current $\left(I_{S}\right)$ :

$$
I_{D S}=I_{S}\left\{i_{f}\left(V_{G B}, V_{S B}\right)-i_{r}\left(V_{G B}, V_{D B}\right)\right\}
$$

In the illustration of the inversion charge distribution in FIG. 3 adopted from [23], one can indicate the corresponding physical section of the channel near the source and drain, which are responsible for the photoresponse with the in situ parameters.

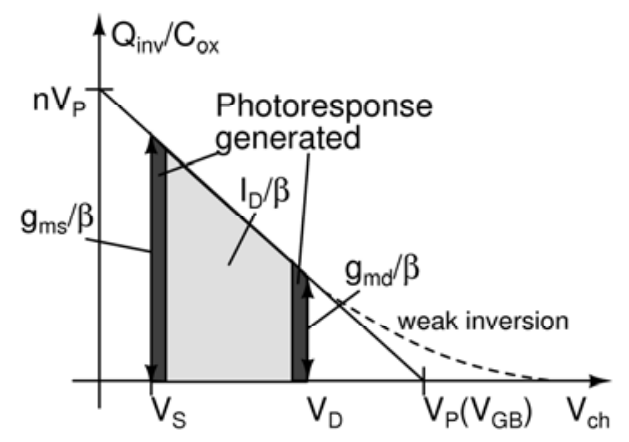

FIG. 3. Illustration of the inversion charge in the channel as a function of the channel potential at $V_{D S}$ voltage. The photoresponse generation is limited to channel sections marked with dark areas, while the source drain current is proportional to the light gray area. $V_{P}$ is the gate bulk dependent pinch-off voltage. 
The Sakowicz et al. [13] model is valid under the condition that out of the plasma perturbation depth, the channel behaves as open boundary. We suppose that as the plasma perturbation depth is smaller than the channel length, the open boundary is preserved for both terminal sides towards the center of the channel. Thus, the $g_{m} / I_{D S}$ can be calculated separately for the affected portions and the photoresponse becomes independent on the source and drain end. This observation gives ground to the followings. The EKV model provides a practical way to find out the independent photoresponse at the ends. If one drives the transistor into saturation $\left(i_{f}=0\right.$ or $\left.i_{r}=0\right)$, the drain potential does not affect the current and the $g_{m} / I_{D S}$ can be estimated by the source side behavior only [26].

$$
\left.V_{r}^{0}\left(V_{G S}\right) \propto \frac{g_{m}}{I_{D S}}\right|_{i_{r}=0} \cong \frac{1}{n} \frac{g_{m s}}{I_{F}}
$$

With other words, the photoresponse $\left(V_{r}\right)$ of a drain side saturated transistor is solely the source side response. The pinch-off (occurs when $V_{D S}>V_{P}$ ) plays an important role, as it indicates the channel potential, which results in zero inversion charge. Thus it eliminates the photoresponse. In the most interesting subthreshold region, the saturation voltage is constant and is about $V_{P}=4 V_{t h} \cong 100 \mathrm{mV}$ in room temperature [24], where $V_{t h}$ is the thermal voltage. Hence, relatively easy to eliminate the drain end photoresponse and get solely the source component in the subthreshold region. The drain side response can be found by using the same process swapping the electronic connectivity of the terminals. Note, that the common approach to determine photoresponse is the open drain configuration by disconnecting the drain from any load. In this situation, the source end conductivity is extended to the complete channel and the response becomes measurable, which is equal to the sought source side response. The measured value is proportional to the intrinsic response, but not equal in case of simultaneous source and drain radiation coupling. 


\section{Nonzero source-bulk potential}

Though the above reasoning was general, it is also worth to examine the practical effect of the source to bulk voltage, $V_{G B}$ to the photoresponse. Principally, $V_{G B}$ alters the pinch-off voltage. With constant $V_{G S}, V_{D S}$ values the $g_{m} / I_{D S}$ ratio slightly changes with $V_{G B}$, namely is proportional to $1 / n\left(V_{G B}\right)$ [23]:

$$
\left.V_{r}^{0}\left(V_{G S}\right) \propto \frac{g_{m}}{I_{D S}}\right|_{i_{r}=0} \cong \frac{1}{n\left(V_{B S}\right) V_{t h}} \frac{1}{\sqrt{i_{f}+0.5 \sqrt{i_{f}}+1}}
$$

The $n\left(V_{G B}\right)$ slope factor can be estimated as:

$$
n \cong 1+\frac{\gamma}{2 \sqrt{V_{P}\left(V_{G B}\right)+2 \phi_{F}}}
$$

where $\gamma$ is the body effect factor and $V_{P}$ is the pinch-off voltage, $\phi_{F}$ is the Fermi level. As the slope factor decreases with $V_{G B}$, and the open drain response is expected to increase by increasing source to bulk potential $\Delta U_{S} \propto 1 / \sqrt{V_{G S}}$. This assumption is verified by sweeping the $V_{G B}$ and $V_{S B}=V_{D B}$ in parallel and the photoresponse is measured. The recorded curves are presented in FIG. 12.

As a conclusion, the photoresponse on the terminals are equal to the intrinsic response at zero source to bulk voltage $\left(V_{r}^{0}\right)$ with the correction of $1 / n\left(V_{G B}\right)$. Due to the device symmetry condition, $V_{r}^{0}\left(V_{G S}\right)=$ $V_{r}^{0}\left(V_{G D}\right)$, we get:

$$
\begin{gathered}
\left.V_{r}\left(V_{G S}\right) \propto \frac{1}{n\left(V_{G B}\right)} \frac{g_{m}}{I_{D S}}\right|_{V_{S}=0}=\frac{1}{n\left(V_{G B}\right)} V_{r}^{0}\left(V_{G S}\right) \\
V_{r}\left(V_{G D}\right) \propto-\left.\frac{1}{n\left(V_{G B}\right)} \frac{g_{m}}{I_{D S}}\right|_{V_{D}=0}=-\frac{1}{n\left(V_{G B}\right)} V_{r}^{0}\left(V_{G S}\right)
\end{gathered}
$$

Combining (12)-(13) with the terminal coupling efficiencies, we conclude the measurable extrinsic photoresponse as a weighted combination of the intrinsic responses: 


$$
\begin{gathered}
V_{r}\left(V_{G S}, V_{G D}\right)=\eta_{S} V_{r}\left(V_{G S}\right)-\eta_{D} V_{r}\left(V_{G D}\right) \\
V_{r}\left(V_{G S}, V_{G D}\right)=\eta_{S} \frac{1}{n\left(V_{G B}\right)} V_{r}^{0}\left(V_{G S}\right)-\eta_{D} \frac{1}{n\left(V_{G B}\right)} V_{r}^{0}\left(V_{G D}\right)
\end{gathered}
$$

This equation describes why the measurable unloaded, grounded source or drain photoresponse is not equal to the intrinsic photoresponse except for ideal isolation. Rewriting (15) with $V_{G S}=V_{G D}, V_{S B}=0$ yields:

$$
\left.u_{r}\right|_{\text {open }}=\left(\eta_{S(D)}-\eta_{D(S)}\right) V_{r}^{0}\left(V_{G S}\right)
$$

This is an important finding, because in case of cross talk due to finite isolation between source and drain $\left(\eta_{S} \eta_{D} \neq 0\right)$ the measured value is smaller from the open drain (source) response alone.

\section{E. Plasma perturbation depth}

The penetration depth or plasma perturbation depth is estimated by hydrofluidic model [1] or telegrapher's equation solution of transmission lines [14]. D. Veksler et al [27] proposed a photoresponse model based on quasi-static unified charge control model, which describes analytically the rectification under DC bias current with respect to the constant DC biased drain-gate coupled AC signal. In an unbiased FET, the RF signal in the channel is estimated to decays exponentially marking a well-defined penetration depth (or effective length) definition as the length where the AC signal amplitude decays to $1 / e$ of its maximum. In the distributed $\mathrm{RC}$ channel model, let resistor per unit length $r_{0}=1 /\left(q \rho_{s} \mu W\right), \mu$ being carrier mobility in the channel, $W$ channel width, and the capacitances per unit length $c_{0}=\varepsilon_{o x} \varepsilon_{0} W / d$, where $d$ is the thickness of the insulator. The penetration depth can be defined in non current biased case under the non-resonant detection assumption neglecting the channel inductance and gate leakage as [14]:

$$
l_{e f f}=\sqrt{2 /\left(\omega_{a c} r_{0} c_{0}\right)}=\sqrt{q d \rho_{s} \mu /\left(\pi \varepsilon_{o x} \varepsilon_{0} f_{a c}\right)}
$$


The AC signal amplitude than decays as $u_{a c}(l)=u_{a c} \exp \left(-l / l_{e f f}\right)$, and the photoresponse builds up with $\propto\left\{1-\exp \left(-2 l / l_{e f f}\right)\right\}$ rate. However, in the current biased channel, the carrier concentration changes along the channel, resulting in a different boundary conditions for the edges. The effective length could be divided into two quantities, namely the source and the saturated drain side. Substituting the carrier concentration (7) to the effective length expression and neglecting the velocity saturation and gate bias-dependent mobility, for two limiting cases of strong inversion $\left(V_{G S}>V_{T}\right), V_{G S}=V_{T}$, and depletion $\left(V_{G S}<V_{T}\right)$ the simplified expressions are the followings:

$$
\begin{gathered}
l_{e f f(S, D)}^{V_{G S}<V_{T}}=\sqrt{\frac{2 \eta V_{t h} \mu}{\omega} \exp \left(\frac{V_{G S}-V_{T}-\alpha V_{S(D)}}{\eta V_{t h}}\right)}[\mathrm{cm}] \\
l_{e f f(S, D)}^{V_{G S}=V_{T}}=\sqrt{\frac{\eta V_{t h} \mu}{\omega}}[\mathrm{cm}] \\
l_{e f f(S, D)}^{V_{G S}>V_{T}}=\sqrt{\frac{\mu}{\omega}\left(V_{G S}-V_{T}-\alpha V_{S(D)}\right)}[\mathrm{cm}]
\end{gathered}
$$

These equations are valid under no current bias, still gives an estimate for the current biased terminal values. The results can be interpreted as a saturated channel FET's source provides deep penetration, while at the drain side the AC signal decays within a fraction of the source side effective length. FIG. 4 shows the evaluated inversion charge distribution (7) and the corresponding AC signal amplitude decay at various source-drain voltage.
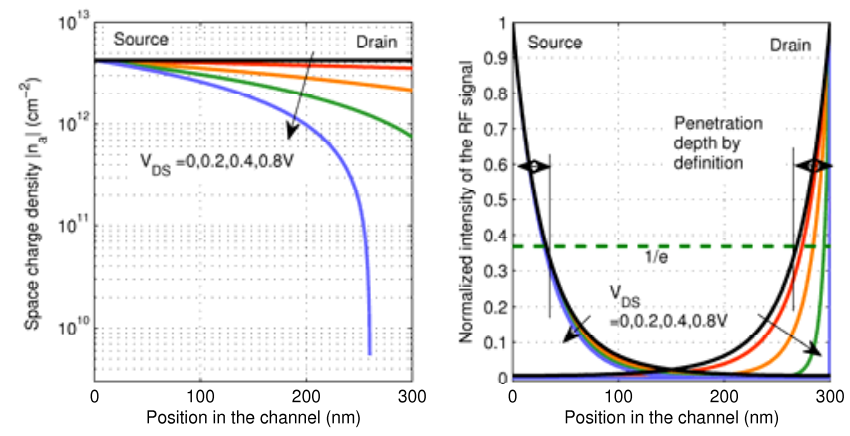
FIG. 4. Space charge density estimation in a current biased FET channel based on the unified charge control model (left) as a function of the $\mathrm{V}_{\mathrm{DS}}$ at typical silicon device parameters as $\mu=330 \mathrm{~cm}^{2} / \mathrm{Vs}, \mathrm{V}_{\mathrm{GS}}=0.5 \mathrm{~V}, \mathrm{~N}_{\mathrm{a}}=10^{16} \mathrm{~cm}^{-3}, \mathrm{~d}_{\mathrm{ox}}=3.2 \mathrm{~nm}, \mathrm{~T}=300 \mathrm{~K}, \mathrm{~L}=300 \mathrm{~nm}, \eta=1.9, \alpha=1$. The normalized source and drain side plasma perturbation decay (right) as a function of the $V_{D S}$ at $f_{R F}=360 \mathrm{GHz}$.

\section{IN CIRCUIT BEHAVIOR}

Placing the detector in a circuit environment, such as resistive load and current source, the measurable value becomes even more diverse. The photoresponse can be measured between the source and drain terminals. In practice, one terminal is set to a fixed potential and the other remains open or loaded with a resistor. The generated response is recorded as voltage using DC or lock-in technique with low frequency modulation. Under source drain current, the detector behaves as a usual circuit element with respect to the rectified $\mathrm{DC}$ or modulated $\mathrm{AC}$ signal. Consequently, the measured values are not the photoresponse solely, but its amplified or attenuated variant (the extrinsic photoresponse), depending on the circuit surroundings. The reported current biased response enhancement and frequent sign change can be described by the classic circuit theory. In order to validate this assumption, we consider four basic arrangement of the detector environment (see FIG. 5).

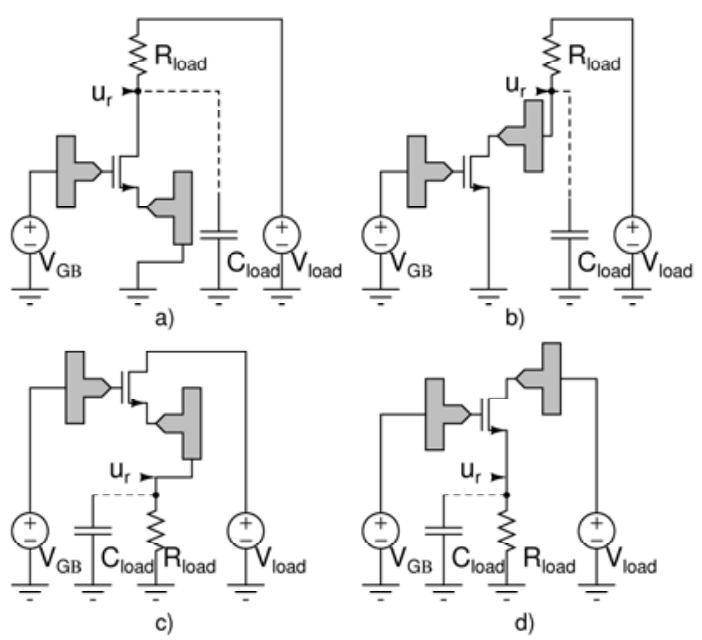

FIG. 5.

The analyzed circuit schemes. The "T" shaped polygons representing the antenna wings. 
Based on the long channel model, the source and drain side photoresponses are modeled by voltage controlled voltage sources in the circuits as shown in FIG. 6 for the different load schemes. Considering single terminal antenna coupling (e.g. source-driven approach), the common ground is that the photoresponse is linked to one terminal (source or drain), regardless of the rectification mechanism.

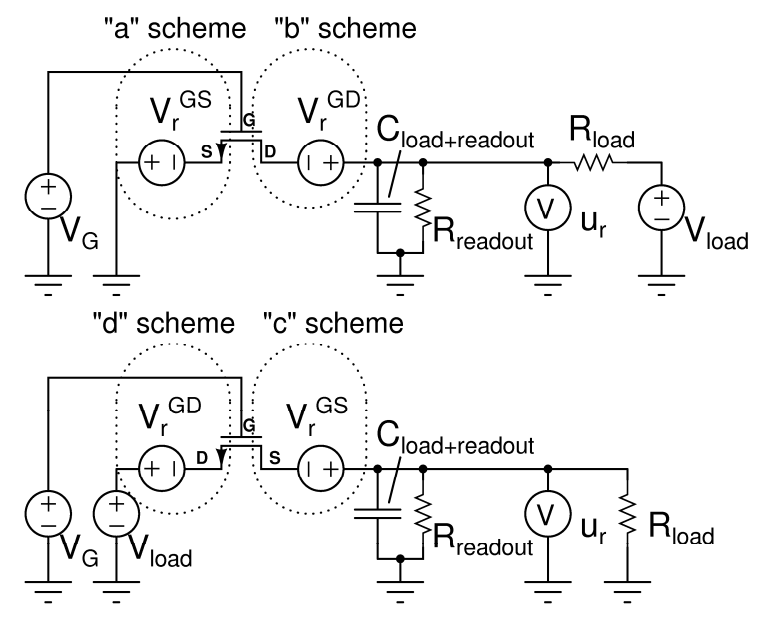

FIG. 6. The circuit arrangements a-d with the rectified photoresponse voltage sources.

Note, that the detector FET becomes a common-gate amplifier ("a" scheme) or a voltage follower ("c" scheme) from the rectified DC or low frequency modulated signal point of view. Depending on the terminal side of the generated photoresponse, the observation point provides different views of the original signal through the amplifiers. One can express the small signal AC relation between the generated signals and the measured signal by the well known $\pi$ FET circuit model for the different cases (see Table I.).

TABLE I. Small signal transfer function of different schemes

Scheme Small signal transfer function

$$
\begin{gathered}
A_{a}=\frac{V_{r}}{V_{r}^{G S}}=-\frac{\left(g_{d s}+g_{m}\right) Z_{\text {load }}}{1+g_{d s} Z_{\text {load }}} \\
\text { "b" } \\
A_{b}=\frac{V_{r}}{V_{r}^{G D}}=\frac{g_{d s} Z_{\text {load }}}{1+g_{d s} Z_{\text {load }}}
\end{gathered}
$$




$$
\begin{gathered}
\hline \hline \text { "c" } A_{c}=\frac{V_{r}}{V_{r}^{G S}}=\frac{\left(g_{d s}+g_{m}\right) Z_{\text {load }}}{1+\left(g_{d s}+g_{m}\right) Z_{\text {load }}} \\
\text { "d" } A_{d}=\frac{V_{r}}{V_{r}^{G D}}=-\frac{g_{d s} Z_{\text {load }}}{1+\left(g_{d s}+g_{m}\right) Z_{\text {load }}} \\
\hline Z_{\text {load }}=1 / j \omega_{m}\left(C_{\text {load }}+C_{\text {readout }}\right) \|\left(R_{\text {load }} \| R_{\text {readout }}\right) \\
\omega_{m} \text { is the modulation frequency. }
\end{gathered}
$$

The actually measurable value $\left(u_{r}\right)$ is a superposition of the amplified photoresponses, because introducing the transfer function into (14) leads to the following formulas for the different schemes:

$$
\begin{aligned}
& u_{r}^{a(b)}=\eta_{S(D)} V_{r}^{G S} A_{a}+\eta_{D(S)} V_{r}^{G D} A_{b} \\
& u_{r}{ }^{c(d)}=\eta_{S(D)} V_{r}^{G S} A_{c}+\eta_{D(S)} V_{r}^{G D} A_{d}
\end{aligned}
$$

The open drain (source) configuration can be reproduced as well in any schemes by $V_{\text {load }}=0, R_{\text {load }} \rightarrow$ $\infty$, which leads to $A_{a, d}=-1, A_{b, c}=1$. Substituting $R_{c h}=1 / g_{d s}$ in (24) and $\eta_{s}=1, \eta_{D}=0$ leads to the load model described in [13]:

$$
u_{r}=V_{r}^{0} \frac{g_{d s} Z_{\text {load }}}{1+g_{d s} Z_{\text {load }}}=V_{r}^{0} \frac{1}{1+R_{C H} / Z_{\text {load }}}
$$

In order to illustrate the importance of the varying characteristics of the circuit behavior, FIG. 7 shows the small signal transfer function of different schemes. The common gate amplifier arrangement amplification $A_{a}$ is the largest, while all other case provides at most gain of unity.
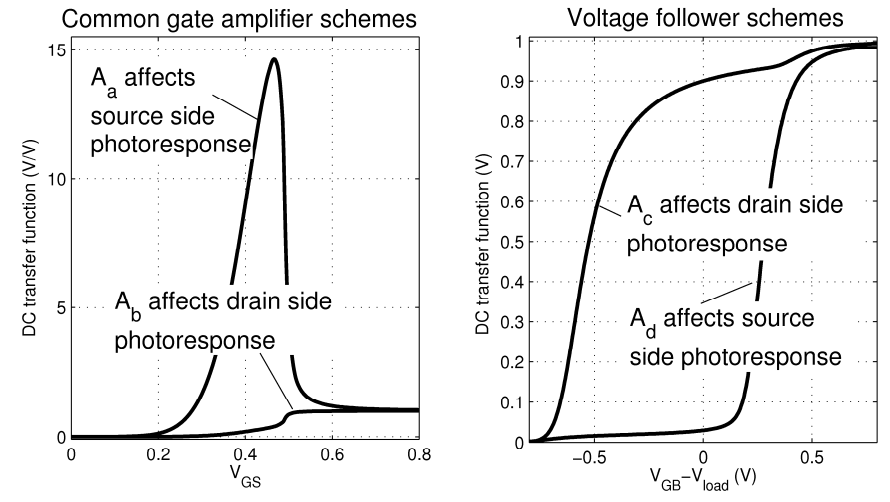
FIG. 7. Illustrative small signal transfer functions for the different schemes. Please note the scale of the curves in different cases.

For a given setup and radiation settings, the $\eta_{S(D, G)}$ cannot be identified independently from each other and from the fitting parameter $k$ of (1) and (2). From practical point of view, the ratio of them is important, that shows if there is any cross talk between them and to what extent. The proposed solution uses the consequence of (9): drive the transistor into saturation and measure source side response only. Then the $\eta_{D} / \eta_{S}$ ratio can be calculated from the measured response ration in saturation:

$$
u_{r}^{b} / u_{r}^{a} \cong \eta_{D} / \eta_{S}
$$

The (14)-(15) expressions suggest that the RF coupling scheme to the terminals and the cross talk between the terminals affects significantly the recorded response. In the subthreshold region, as the transistor reaches the saturation, $g_{m} \gg g_{d s}$, and the difference between the source and drain coupled cases becomes notable. The source coupled scheme under source drain current is expected to response with high sensitivity increase, while the drain coupled one shall response with small value dominated by the source-drain cross talk. At higher gate voltages the two cases becomes similar again when $g_{d s}$ governs the transfer functions $\left(\left|A_{a-d}\right| \rightarrow 1\right)$. In the transition to saturation $\left(g_{m} \approx g_{d s}\right)$, the two terminal's RF opposite sign response is summed with varying weights, hence response sign change may occur. In the subsequent measurements, all of these situations are investigated. FIG. 8 illustrates the model operation. 

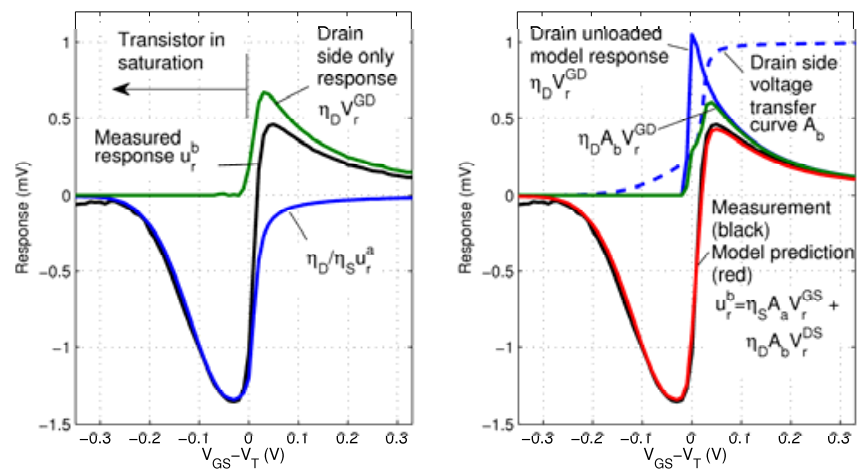

FIG. 8 .

(Color online) Illustration how the model describes the measured response (right) and estimates the source drain simultaneous radiation coupling with its ratio based on saturated region measurement (left).

\section{EXPERIMENTS}

In the model validation two samples have been measured. The first is a commercial GaAs pHEMT and the second is a custom designed silicon detector. The relatively large pHEMT enabled to focus the radiation yielding different $\eta_{S}, \eta_{D}$ weights on the source and drain terminals, while the silicon sample is useful to analyze the source to bulk effect. The applied radiation was $f_{R F}=358 \mathrm{GHz}$ generated by a VDI CW upconversion source. The source had $0.8 \mathrm{~mW}$ output power. The readout circuit had the following parameters: the modulation frequency was $\omega_{m}=2 \mathrm{KHz}, R_{\text {load }}=1 \mathrm{M} \Omega, R_{\text {readout }}>2 G \Omega, C_{\text {load }}+$ $C_{\text {readout }} \cong 130 p F$

\section{F. GaAs pHEMT sample}

The sample was an Avago Technologies ATF-36077 GaAs pHEMT. The device has a nominal $L=$ $200 \mathrm{~nm}$ gate length with a total gate width of $W=200 \mu \mathrm{m}$. The effective signal generation length is estimated by (19) as $l_{\text {eff }} \approx 241 \mathrm{~nm}$ with $\mu_{G a A s} \cong 8000 \mathrm{~cm}^{2} / V s$, hence the model is valid in subthreshold. During the measurements, the transistor is connected in "a" and "b" schemes. Both source and drain side in-circuit $\left(A_{a, b}\right)$ and open drain AC transfer characteristics $\left(A_{a, b}^{o p e n}\right)$ are measured along with the zero current response. The radiation was focused by off-axis parabola mirrors: first near the 
gate-source pads and then in the middle of the device. According to the described method, the $\eta_{S} / \eta_{D}$ ratio is calculated based on (24) from two measurements in saturation mode: one at the source (a) and one at the drain side (b), respectively. As a next step the open drain response has to be determined. It is not calculated, due to lack of device model, but derived from measurement. It turned out, that the open drain (source) characteristics is seriously degraded by the limiting open drain AC transfer function. In order to get a more realistic intrinsic response $V_{r}^{0}$, the inverse function of the corresponding AC transfer $\left(A^{\text {open }}\right)$ is applied to the measured response $u_{r}^{\text {open }}$ as: $V_{r}^{0}=\eta_{S} / \eta_{D} A^{\text {open-1}}\left(u_{r}^{\text {open }}\right)$. This transfer function can be determined by small signal AC measurements. FIG. 9 and FIG. 10 show the recorded responses and the model predictions. As expected in the gate-source side focus, the drain side responded with smaller intensity $\eta_{S} / \eta_{D} \cong 11 / 1$, and the middle focus situation yields higher overlap $\eta_{S} / \eta_{D} \cong$ 2.3/1. The curves in FIG. 9 are normalized to the maximum measured open drain response. Worth to note, that the apparent amplifications of the bias current are different in the two cases. The reason is principally that the measured $u_{r}^{o p e n}$ values are the superposition of the drain and source side response with opposite sign. In saturation $\left(V_{G S}<-0.7 V\right)$, the source side operates only, hence seems to be larger compared to the decreased open drain response. As a limiting case, when the source and drain side has equal radiation, the open drain response disappears, the current biased case remains near unchanged and the apparent amplification is extreme. 


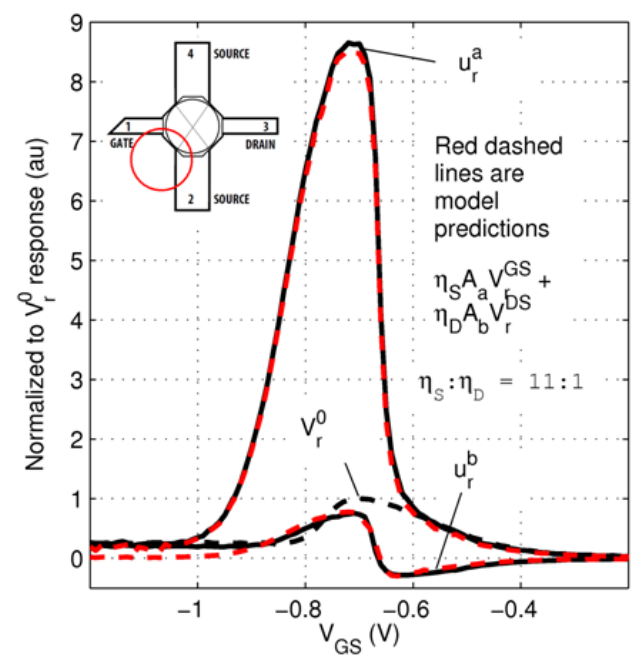

FIG. 9. (Color online) Measurement and model prediction results of the pHEMT at 320 GHz. The insets show the approximate focus point of the radiation, which is located at the source-gate region. $\omega_{m}=2 \mathrm{KHz}, R_{\text {load }}=1 M \Omega, V_{\text {load }}=2 \mathrm{~V}$, the $I_{D S} \cong 0 \ldots 2 \mu A m p$. The results shows a 11:1 ratio between the source and drain side response.

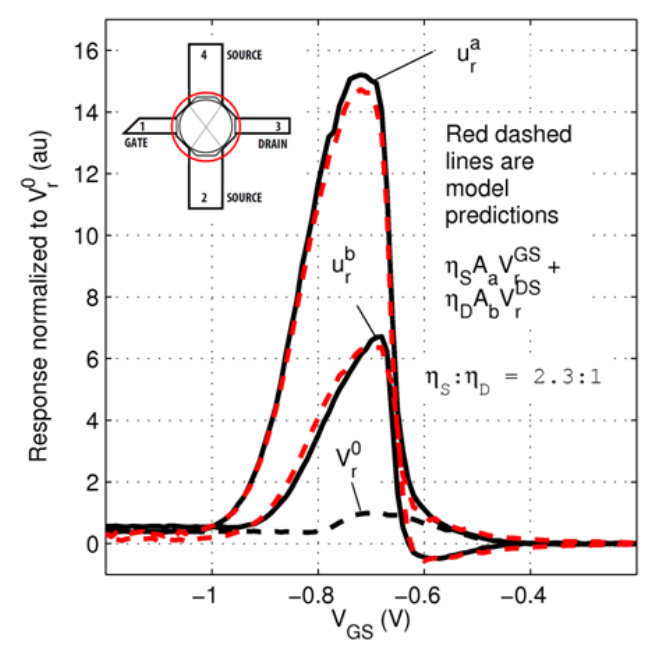

FIG. 10. (Color online) Measurement and model prediction results of the pHEMT at 320 GHz. The radiation is focused in the middle of the device. $\omega_{m}=2 \mathrm{KHz}, R_{\text {load }}=1 M \Omega, V_{\text {load }}=2 \mathrm{~V}$, the $I_{D S} \cong 0 \ldots 2 \mu \mathrm{Amp}$. The results shows a $2.3: 1$ ratio between the source and drain side response.

\section{G. Silicon sample}

The silicon sample is an antenna coupled transistor design that has been manufactured in standard 180 $\mathrm{nm}$ technology. The microphoto and illustrative cross section can be seen in FIG. 11. In the detector 
chip, a "H" shaped dipole antenna has been implemented as well. This antenna-detector have been selected as all of its terminals are connected to external pads. The gate and one terminal of the transistor are coupled this way to the antenna, while the other terminal is connected to a pad directly. The antenna wings are also routed to pads as well. Its resonant peak was $358 \mathrm{GHz}$, hence, the measurements have been performed at this frequency. In the center of the dipole, using a staircase via series, a $W=$ $440 \mathrm{n}, L=300 \mathrm{~nm}$ drawn gate sized NMOS is coupled to the antenna wings. The effective signal generation length by (19) is $l_{\text {eff }} \approx 49 \mathrm{~nm}$ at $\mu_{S i} \cong 330 \mathrm{~cm}^{2} / V s$, thus $l \ll L$ and the model holds. The incident RF power was $10 \mathrm{uW}$ on the effective area of the antenna and the responsivity was $\sim 100 \mathrm{~V} / \mathrm{W}$.

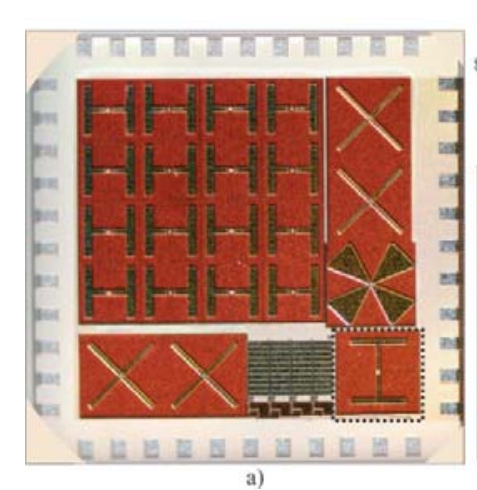

a)

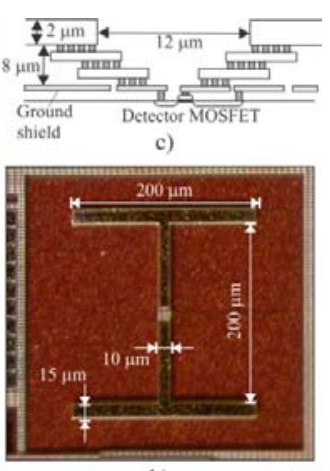

b)

FIG. 11. (Color online) Mircophoto a) of the ASIC used in the measurements. The b) is the antenna coupled detector and the top inset illustrates the cross-section of the near detector antenna structure.

The chip and the source are aligned to match radiation polarity and the chip is placed in front of the source in its farfield without focusing. First, the source to bulk dependency was examined. The $V_{G B}$ is swept, while the $V_{D S}=0 \mathrm{~V}$ was maintained by applying increasing $V_{S B}=V_{D B}$ values with $R_{\text {load }}=1 \mathrm{M} \Omega$ and $\omega_{m}=2 \mathrm{KHz}$. The expected increasing response (equ 10) can be seen in FIG. 12. 


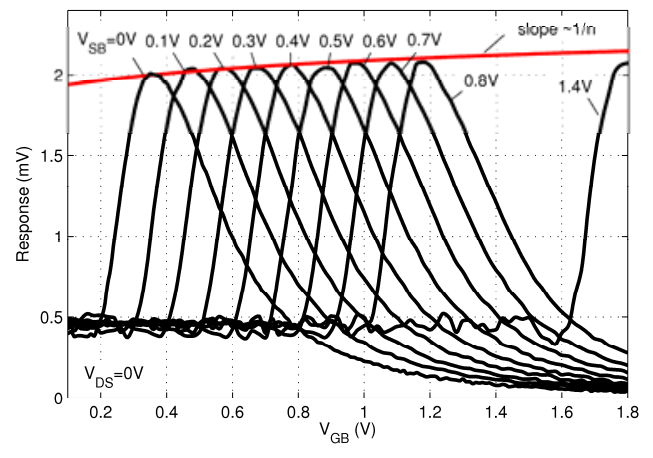

FIG. 12. (Color online) The effect of the source-to-bulk potential to the open drain photoresponse. $V_{D S}=0 \mathrm{~V}, V_{S B}=V_{D B}, \omega_{m}=2 \mathrm{KHz}$.

Then, the source and drain coupling schemes are measured by flipping the external components according to FIG. 5. As the antenna structure is fixed and the radiation wavefront is set to near flat, the source-gate and drain-gate coupling was fixed. According to the method it was calculated as $\eta_{S} / \eta_{D} \cong$ 6.13/1. The measurement results and modeled photoresponse are presented in FIG. 13 for "a" and "b" schemes, and in FIG. 14 for "c" and "d" schemes. In the later case, the $V_{\text {load }}$ has significant effect on the measured response, as the transistor went from saturation to linear region depending on the load potential.
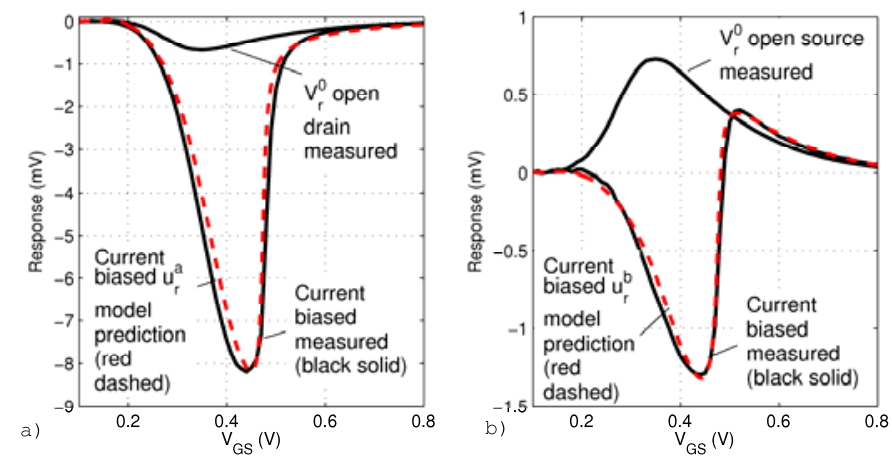

FIG. 13. (Color online) Measured and modeled photoresponse for the a) "a" scheme and b) "b" scheme at $I_{D S}=0, V_{\text {load }}=0 \mathrm{~V}, \omega_{m}=2 \mathrm{KHz}$ and $\omega_{m}=2 \mathrm{KHz}, R_{\text {load }}=1 \mathrm{M} \Omega, V_{\text {load }}=1.8 \mathrm{~V}$, the $I_{D S} \cong 0 \ldots 1.8 \mu \mathrm{Amp}$. 


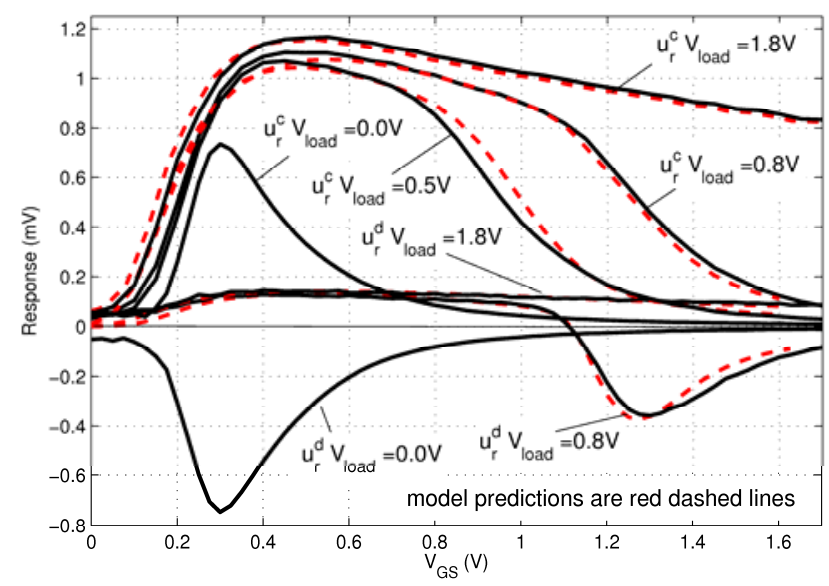

FIG. 14. (Color online) Measured and modeled photoresponse for the "c" and "d" schemes with different $V_{\text {load }}$ and $\omega_{m}=2 \mathrm{KHz}$ and $R_{\text {load }}=$ $1 M \Omega, V_{\text {load }}=0 ; 0.5 ; 0.8 ; 1.8 \mathrm{~V}$.

Finally, the resistive load has been replaced by a current source. The corresponding "a" scheme and b) "b" scheme results are presented in FIG. 15.
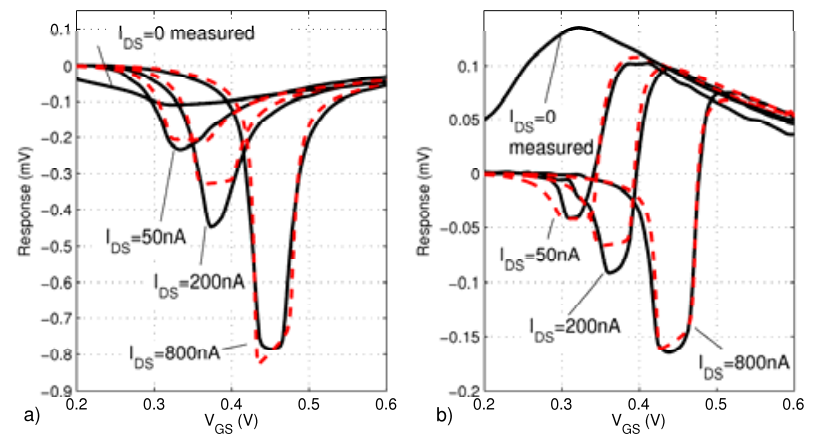

FIG. 15. (Color online) Measured and modeled photoresponse for the a) "a" scheme and b) "b" scheme with current generator load instead of resistive load at $\omega_{m}=2 \mathrm{KHz}$.

\section{DISCUSSION}

The good matching of the model with the measurements suggests that the photoresponse is not altered by the current flow. The importance is emphasized of separating the radiation generated intrinsic response from the actually measured extrinsic value. In case of cross talk due to finite isolation between 
source and drain $\left(\eta_{S} \eta_{D} \neq 0\right)$ the measurable value is smaller from the open drain (source) response alone.

It is also the result of finite isolation that the current biased amplification becomes misleading. If the measured photoresponse differs with swapped source and drain terminals and/or the response sign change a definite sign of finite isolation. In order to separate the source and drain end response, and to find out the ratio of them, we described a simple method: biasing the transistor in saturation and then swapping the electronic connectivity of the two terminals. The ratio of the measured values gives the ratio of the source drain coupling. By applying the inverse of the AC small signal transfer characteristics to the measurement results, the real unloaded photoresponse can be derived as well.

During circuit design, the $g_{m} / I_{D}$ ratio is easy to simulate, a good indicator of the expected photoresponse. A voltage controlled voltage source containing a lookup table (similar to [28]) of the photoresponse can be inserted into any circuit. The small signal behavior is also straightforward to handle, hence a complex circuit can be designed involving FET detectors. However, the $g_{m} / I_{D}$ model overestimates the response in strong inversion in the silicon sample. The reason could be that in strong inversion the $C_{G S}$ and $C_{G D}$ becomes much larger than in weak inversion or in depletion. These may constitute a coupling between the terminals and alter the $\eta_{S} / \eta_{D}$ ratio. Important to note, that the sourcegate, drain-gate capacitance calculation as the base of the current biased description cannot be used in the long channel case as suggested in [1]. The reason is that the Meyer's capacitance model or the more precise EKV and other models always describe the whole channel charge distribution and relies on charge sharing between the source and the drain. On the contrary, the charge density responsible for the photoresponse is limited into a small portion of the channel. Hence, the integral type capacitance models do not explain these portions only, and cannot be used for precise response predictions. 
The noise performance (signal-to-noise ratio) is also could be incorporated in an electronic simulation as the important noise sources are modeled in various electronic simulation model. From theoretical point of view, as the photoresponse does not altered by the bias current, the appearing current induced noise phenomena will decrease the SNR. Hence, the achievable SNR can be estimated by the noise factor (NF) of the circuit schemes (NF is the ratio of the output and SNR and measures of degradation of the SNR). For the common gate amplifier scheme the minimal $N F \approx 1.6-3$ depending on the technology and transistor parameters. It means that the SNR will decrease at least by this amount. This estimate may describe the experimental results measured in A. Lisauskas el at. [5].

Though the model is introduced for long channel samples, it is expected that the extrinsic behavior of the transistors does not change significantly if the photoresponse builds up throughout the complete channel as the HEMT sample demonstrated. The apparent amplification and the other statements hold with corrections to the distributed response generation, and the difference between the source and drain coupling vanishes as the whole channel becomes active.

\section{ACKNOWLEDGMENT}

The support of the grants TÁMOP-4.2.1.B-11/2/KMR-2011-0002 and TÁMOP-4.2.2/B-10/1-2010-0014 is gratefully acknowledged. I also thank the work of Domonkos Gergely and Zsolt Benedek in the layout design of the detector chip.

\section{REFERENCES}

[1] D Veksler et al., "Detection of terahertz radiation in gated two-dimensional structures governed by dc current," Physical Review B, vol. 73, no. 12, p. 125328, 2006.

[2] Michel Dyakonov and Michael S Shur, "Current instability and plasma waves generation in ungated two-dimensional electron layers," Applied Physics Letters, vol. 87, no. 11, pp. 111501--111501, 
2005.

[3] JQ Lu and MS Shur, "Terahertz Detection by High Electron Mobility Transistor: Effect of Drain Current," in Twelfth International Symposium on Space Terahertz Technology, vol. 1, 2001, p. 103.

[4] TA Elkhatib et al., "Terahertz response of field-effect transistors in saturation regime," Applied Physics Letters, vol. 98, no. 24, pp. 243505--243505, 2011.

[5] Alvydas Lisauskas et al., "Terahertz responsivity and low-frequency noise in biased silicon fieldeffect transistors," Applied Physics Letters, vol. 102, no. 15, pp. 153505--153505, 2013.

[6] VV Popov, MS Shur, GM Tsymbalov, and DV Fateev, "Higher-order plasmon resonances in GaNbased field-effect transistor arrays," International Journal of High Speed Electronics and Systems, vol. 17, no. 03, pp. 557--566, 2007.

[7] DB Veksler et al., "Imaging of field-effect transistors by focused terahertz radiation," Solid-State Electronics, vol. 53, no. 6, pp. 571--573, 2009.

[8] Erik Ojefors, Ullrich R Pfeiffer, Alvydas Lisauskas, and Hartmut G Roskos, "A 0.65 THz focal-plane array in a quarter-micron CMOS process technology," Solid-State Circuits, IEEE Journal of, vol. 44, no. 7, pp. 1968--1976, 2009.

[9] Hani Sherry et al., "A 1kpixel CMOS camera chip for 25fps real-time terahertz imaging applications," in Solid-State Circuits Conference Digest of Technical Papers (ISSCC), 2012 IEEE International, 2012, pp. 252--254.

[10] A Lisauskas, D Glaab, HG Roskos, E, and UR Pfeiffer, "Terahertz imaging with Si MOSFET focalplane arrays," in Proc. SPIE, vol. 7215, 2009, p. 72150J.

[11] I Stanley, "A tutorial review of techniques for coherent optical fiber transmission systems," Communications Magazine, IEEE, vol. 23, no. 8, pp. 37--53, 1985.

[12] Michael Dyakonov and Michael Shur, "Shallow water analogy for a ballistic field effect transistor: New mechanism of plasma wave generation by dc current," Physical review letters, vol. 71, no. 15, pp. 2465--2468, 1993.

[13] M Sakowicz et al., "Terahertz responsivity of field effect transistors versus their static channel conductivity and loading effects," Journal of Applied Physics, vol. 110, no. 5, pp. 054512--054512, 2011. 
[14] S Preu et al., "An improved model for non-resonant terahertz detection in field-effect transistors," Journal of Applied Physics, vol. 111, no. 2, pp. 024502--024502, 2012.

[15] A. Gutin, T. Ytterdal, V. Kachorovskii, A. Muraviev, and M. Shur, "THz SPICE for Modeling Detectors and Nonquadratic Response at Large Input Signal," Sensors Journal, IEEE, vol. 13, no. 1, pp. 55-62, 2013.

[16] Naoki Oda et al., "Development of terahertz focal plane arrays and handy camera," in SPIE Defense, Security, and Sensing, 2011, pp. 80121B--80121B.

[17] Jonathan Y Suen et al., "Towards medical terahertz sensing of skin hydration," Stud. Health Technol. Inform, vol. 142, pp. 364--368, 2009.

[18] Richard Al Hadi et al., "A broadband 0.6 to 1 THz CMOS imaging detector with an integrated lens," in Microwave Symposium Digest (MTT), 2011 IEEE MTT-S International, 2011, pp. 1--4.

[19] Sebastian Boppel et al., "CMOS Integrated Antenna-Coupled Field-Effect Transistors for the Detection of Radiation From 0.2 to $4.3 \mathrm{THz}$," IEEE transactions on microwave theory and techniques, vol. 60, no. 12, pp. 3834--3843, 2012.

[20] Peter Foldesy, "Characterization of silicon field effect transistor sub-THz detectors for imaging systems," in Circuits and Systems (ISCAS), 2012 IEEE International Symposium on, 2012, pp. 934-937.

[21] F., D. Flandre, and P. G. A. Jespers Silveira, "A gm/ID based methodology for the design of CMOS analog circuits and its application to the synthesis of a silicon-on-insulator micropower OTA.," IEEE Journal ofSolid-State Circuits, vol. 31, no. 9, pp. 1314-1319, 1996.

[22] Eric A. Vittoz, "The Fundamentals of Analog Micropower Design," in Circuits and systems tutorials.: John Wiley and Sons, 1996, pp. 365-372.

[23] Eric A. Vittoz Christian C. Enz, Charge-Based MOS Transistor Modeling: The EKV Model for LowPower and RF IC Design.: John Wiley \& Sons, 2006.

[24] A.B. Bhattacharyya, Compact MOSFET models.: John Wiley \& Sons, 2009.

[25] Fjeldly A. Tor, Ytterdal Trond, and Shur Michael, Introduction to Device Modeling and Circuit Simulation. New York: John Wiley \& Sons, 1998.

[26] Marcio Cherem Schneider Carlos Galup-Montoro, Mosfet Modeling for Circuit Analysis And 
Design.: World Scientific, 2007.

[27] W Knap et al., "Nonresonant detection of terahertz radiation in field effect transistors," Journal of Applied Physics, vol. 91, no. 11, pp. 9346--9353, 2002.

[28] Monica Fernandez-Barciela et al., "A simplified broad-band large-signal nonquasi-static tablebased FET model," Microwave Theory and Techniques, IEEE Transactions on, vol. 48, no. 3, pp. 395--405, 2000. 